\title{
Elbow pain in pediatrics
}

\author{
Marshall Crowther
}

Published online: 14 March 2009

(c) The Author(s) 2009. This article is published with open access at Springerlink.com

\begin{abstract}
The pediatric and adolescent elbow is subject to both acute and chronic overuse injuries. The practitioner should develop a classification system to evaluate all such injuries, with first focusing on whether the injury represents an acute episode or rather it represents a more chronic problem. In addition, localizing the area of pain as being either medial, lateral, or posterior can better help differentiate the diagnosis. Youth baseball pitchers and throwers are particularly at risk for overuse injuries of the elbow, most of which are related to an injury mechanism termed "valgus extension overload". The most common entity related to this is termed "Little Leaguer's Elbow." Treatment is usually conservative, but for some injuries surgery may be required, especially for displaced medial epicondylar avulsion fractures. Other acute injuries also should be easily recognizable by the general clinician including annular ligament displacement (nursemaid's elbow) which represents one of the most common upper extremity injuries presenting to emergency rooms in youngsters under the age of 6 . Most studies seem to indicate a hyperpronation reduction technique may be more successful then the flexion/supination technique. It is also important to have an awareness of some of the common elbow fractures seen in the younger patient, in particularly supracondylar fractures owing to their high propensity for complications. When
\end{abstract}

M. Crowther

American Sports Medicine Institute (ASMI), Birmingham, AL, USA

Present Address:

M. Crowther $(\square)$

UAB Sports Medicine, 1600 7th Ave South, ACC Suite 402,

Birmingham, AL 35233, USA

e-mail: marshall.crowther@gmail.com;

marshall.crowther@ortho.uab.edu evaluating the elbow for fractures, it is necessary to have an understanding of the appearance of the ossification centers seen on the pediatric elbow.

Keywords Pediatric elbow · Little leaguer's elbow; supracondylar fracture · Valgus extension overload $\cdot$ Annular ligament displacement

It is important to recognize and appropriately manage the common causes of elbow pain seen in pediatric and adolescent patients. A classification system should be used in order to appropriately consider all diagnostic possibilities. First, determining the acuity and chronicity of the pain is important. If the complaint is of an acute nature or related to injury, further delineating the exact mechanism of injury can also be of help in making a presumptive differential diagnosis. Finally, categorizing the location of pain as medial, lateral, posterior, or anterior can further narrow the diagnostic possibilities.

A majority of the causes of elbow pain in the growing youth will be related to overuse injuries and poor mechanics of the immature skeleton. Overhead throwing, and baseball pitching in particular, is a common cause for these overuse injuries in young individuals and thus having an understanding of the biomechanics involved with the overhead throwing motion will allow for clearer conceptualization of how these problems present and how they may be prevented. The most common of these overuse injury patterns are related to a mechanism know as "valgus extension overload" [1]. During the early phases of throwing, the elbow has a medially-sided tension force along with a lateral compressive force which invariably led to specific injury patterns. In addition, during the late stages of throwing, the elbow becomes locked in full extension resulting in 
increased stress of the posterior structures, including the olecranon and olecranon fossa.

In addition to elbow pain secondary to chronic, overuse type injuries, practitioners must be aware of some of the more important acute traumas that can lead to elbow pain, including some of the common fractures that are seen in the pediatric elbow. Most important of these fractures are supracondylar fractures, owing to the fact that they are associated with higher risk of malunion and neurovascular compromise. Nursemaid's elbow, or annular ligament displacement, is a common cause of elbow pain and decreased elbow use in children less than 6-year-old.

\section{Medial elbow pain}

As mentioned earlier, during the early phases of throwing the medial elbow structures are subjected to medial tensile forces. This leads to the common overuse syndromes presenting with medial elbow pain including medial epicondylar aphophysitis, medial epicondyle avulsions, ulnar collateral ligament sprains/tears, and common wrist flexor/pronator strains. The spectrum of these disorders have been given the general term of "Little Leaguer's Elbow" [2]. The medial epicondyle is the last physis of the elbow to close, and both the common wrist flexors/pronators and proximal ulnar collateral ligament originate at this epiphysis.

Clinically, patients with "Little Leaguer's Elbow" will present with progressive medial elbow pain, diminished throwing effectiveness, and loss of throwing velocity. Physical exam findings usually will show tenderness to palpation over the medial epicondyle, with increasing pain noted with resisted wrist flexion. A slight flexure contracture may also be present. Conventional radiographs are the initial imaging test of choice, which may show irregularity and fragmentation of the medial epicondyle. Comparison views of the contralateral elbow should be obtained which may show a widening of the physeal line on the affected side.

Treatment is almost always conservative, with a period of rest with no throwing recommended for 4-6 weeks. Ice and NSAIDs also may be helpful. Elbow and shoulder strengthening exercises and physical therapy are also recommended, followed by a slow interval return to throwing program.

Along the same continuum of medial elbow overuse injury, frank avulsions of the medical epicondyle may occur. The physis is the weak link in the immature skeleton explaining why this injury is much more common than ulnar collateral ligament tears in the younger patients. For non-displaced fractures, a period of rest from throwing is always required, usually 3 months, along with protection from valgus forces and resisted wrist flexion [1]. A short period of immobilization (1-3 weeks) may also be necessary with early ROM exercises [1]. Other strengthening exercises should be started, followed by a slow interval throwing program at the end of the rest period.

Treatment of displaced medial epicondylar fractures tends to be more controversial, but most feel that displacement $>5 \mathrm{~mm}$ requires surgical fixation. Minimally displaced fragments may heal with solid bone union, but sometimes healing only occurs by fibrous union formation. This may be associated with chronic pain and dysfunction in high level throwers [1]. In our practice at Andrews Sports Medicine and Orthopaedic Center, any pitcher or overhead athlete who has even minimal displacement usually gets surgical fixation secondary to the fear that these athletes will not be able to return to full activities with conservative management. A retrospective study is currently being conducted to better answer the question of the need to fix all minimally displaced medial epicondylar physeal fractures.

\section{Lateral elbow pain}

It is important to be aware of two major causes of lateral elbow pain seen in pediatric and adolescent patients. Osteochondritis dessicans of the Capitellum and Panner's Disease both can present with lateral pain, and are differentiated by the age of presentation, etiology, and radiographic findings.

The cause of OCD of the capitellum has largely been unknown and controversial, but many feel it is related to the combination of repetitive microtrauma across the radiocapitellar joint and the tenuous blood supply of the capitellum [3]. Baseball pitchers and gymnasts seem to be most prone to these high stresses across the radio-capitellar joint [6]. In baseball pitching, compression and shear forces occur at the radio-capitellar joint during the late cocking phase. In gymnasts, $60 \%$ of the force across the elbow joint when in full extension occurs at the radio-capitellar joint [4]. In addition to these increased forces seen in active athletes, the radial head cartilage seems to be stiffer than the cartilage of the lateral capitellum, thus creating a mechanical mismatch, further leading to capitellar injury [5]. The tenuous blood supply of the capitellar epiphysis is a result of the lack of humeral metaphyseal collateral circulation meaning that the capitellar epiphysis receives its entire blood supply from one or two isolated posterior vessels that must transverse the entire cartilaginous capitellum [7]. Because of this, the ability for the capitellar epiphysis to heal itself after episodes of microtrauma is compromised, possibly leading to osteonecrosis and eventually an OCD lesion. 
OCD of the capitellum usually presents in the 11- to 15year-old adolescent, and baseball pitchers and gymnasts are the most commonly affected persons. Patients may have several months of pain prior to seeking medical attention. A total of $90 \%$ of patients will complain of pain localized on the lateral aspect of the elbow, but some may have diffuse pain, or no pain at all [1]. The pain typically is relieved by rest and not associated with any nocturnal symptoms. Other symptoms may include loss of motion (usually extension), locking, or catching. If the latter two features are present, one must consider the possibility that articular cartilage fragmentation and resulting loose body formation has occurred [3].

Physical exam findings may reveal tenderness to palpation over the radial capitellar joint and occasionally loss of extension. They may also have a positive "active radiocapitellar compression test" which is considered positive if the patient's pain is reproduced as they actively pronate and supinate their forearm with the elbow locked in full extension while applying a valgus force [3].

Conventional radiographs are the preferred initial diagnostic test of choice when evaluating for capitellar OCD lesions. AP views of the elbow in $45^{\circ}$ of flexion may be the most helpful in identifying the abnormalities associated with this lesion [8]. Flattening and rarefaction of the capitellar subchondral bone can be seen. MRI may show earlier changes that may not yet be visible on plain radiographs, and the earliest change may be seen as low signal intensity at the capitellar surface on T1-weighted images only [8]. MR arthrography may be better at assessing fragment stability.

Treatment options have been somewhat controversial. Non-operative treatment is usually reserved for early lesions without mechanical symptoms or obvious loose bodies. If the overlying cartilage is still intact, these tend to be managed best conservatively. Patients should be removed from the inciting trauma (pitching, gymnastics) for at least 6 months, and usually pitchers should not return to this position in the future. Once symptoms have resolved, strengthening exercises may be started. A total of $50 \%$ of lesions tend to heal non-operatively [3]. Surgical intervention usually is reserved for patients who fail to respond to 6 months of conservative treatment, or those who have mechanical symptoms, unstable fragments, or obvious loose bodies [6]. Surgical options may include arthroscopic loose body removal, debridement of lesion with abrasion chondroplasty, osteochondral autologous transplantation, or rarely fragment reimplantation. Despite treatment, about half of patients may have long term sequelae, including degenerative joint disease [3].

Panner's Disease affects younger patients than those typically with OCD lesions, usually in the age group of 712 years. It represents an avascular necrosis of the capitellum, similar to the other pediatric osteochondroses including Legg-Calve-Perthes disease. In contrast to Osteochondritis dessicans, it is not associated with repetitive trauma and the capitellum typically reconstitutes with time without any associated long term sequelae. Conventional radiographs usually show flattening and patchy sclerosis of the entire capitellum. Treatment is conservative, with restriction from aggravating activities, including those involving axial loading such as throwing and gymnastics [6].

\section{Posterior elbow pain}

Common causes of posterior elbow pain in the young patients include olecranon apophysitis or avulsion, olecranon bursitis, posterior olecranon impingement, or olecranon osteochondrosis. Repetitive forced extension as well as locking the elbow in full extension, which are commonly seen in the follow-through/deceleration phases of pitching, can lead to traction across the olecranon apophyis where the triceps tendon inserts, resulting in olecranon apophysitis [1]. Frank avulsions can also occur, but they usually preceded the chronic posterior elbow pain indicative of ongoing olecranon apophysitis. Physical exam findings usually show tenderness over the olecranon apophysis. Conventional radiographs can demonstrate physeal irregularity, fragmentation, widening, or frank avulsion. Again comparison views of the contralateral elbow are imperative for comparison. Treatment for olecranon apophysitis and non-displaced physeal fractures involves rest and suspension of those overhead activities such as throwing that cause repetitive extension and forced hyperextension at the elbow. Throwing athletes typically may need 2-3 months of rest to decrease risk of reoccurrence. For displaced fractures, surgical fixation is usually required, and may hasten time to return to sport [1].

Olecranon bursitis can also occur in young athletes secondary to acute or chronic trauma occurring over the dorsal aspect of the elbow. Physical exam findings usually show significant soft tissue swelling and tenderness posteriorly. The presence of extensive erythema, tenderness, or systemic symptoms should alert the clinician to the possibility of a co-existing cellulitis. Olecranon bursitis can usually be managed conservatively with rest, ice, NSAIDs, compression wrappings, and even elbow pads to allow return to play [1]. If these methods are unsuccessful, aspiration and subsequent corticosteroid into the bursa can be considered. In rare cases, bursectomy may need to be performed [1].

If a throwing athlete presents with posterior elbow pain, and no pain over the olecranon apophysis or significant soft tissue swelling is evident, one should consider the 
possibility of posterior olecranon impingement (i.e., valgus extension overload syndrome). In addition to pain, they may complain of loss of full extension. Physical exam findings include a positive "valgus extension overload" test, in which pain is reproduced when a valgus force is applied at the elbow joint while the elbow is passively forced into maximal extension. The impingement may be caused by medial olecranon osteophytes or intra-articular loose bodies. Symptoms of locking may indicate a foreign body, which may also be seen on radiographs. Treatment initially may consist of physical therapy aimed at improving extension range of motion, but if a loose body is present, arthroscopic removal is usually necessary. Arthroscopic debridement of a medial olecranon osteophyte can also be done if necessary. The presence of posterior olecranon impingement is usually secondary to valgus extension overload, which should alert the clinician to the possibility of an underlying ulnar collateral ligament injury or insufficiency and thus needs thorough evaluation [1].

\section{Elbow fractures}

Elbow fractures are relatively common in children, accounting for at least $10 \%$ of all pediatric fractures [9]. Elbow fractures are often more likely to require reduction than some of the other common pediatric fractures, and can be associated with a higher complication rate [10]. Physical exam and plain radiographs are usually sufficient for accurate diagnosis. However, conventional radiographs of the elbow in the skeletally immature individual can be sometimes difficult to evaluate owing to the secondary ossification centers that may be mistaken for fractures. It is important for providers who care for these injuries be aware of the normal order of appearance of the six secondary ossification centers. This is best accomplished by knowing the pneumonic CRMTOL, which is illustrated in the chart below (Table 1).

Of the elbow fractures, supracondylar fractures are the most common and important to be aware of owing to a high rate of malunion and complications such as neurovascular

Table 1

\begin{tabular}{lll}
\hline & Ossification center & $\begin{array}{l}\text { Age at } \\
\text { appearance (years) }\end{array}$ \\
\hline $\mathrm{C}$ & Capitellum & 1 \\
$\mathrm{R}$ & Radius & 3 \\
$\mathrm{M}$ & Medial epicondyle & 5 \\
$\mathrm{~T}$ & Trochlea & 7 \\
$\mathrm{O}$ & Olecranon & 9 \\
$\mathrm{~L}$ & Lateral epicondyle & 11 \\
\hline
\end{tabular}

injury or compartment syndrome. A total of $60-80 \%$ of elbow fractures are supracondylar [11]. These are usually seen in children during the first decade of life (most common ages 5-7 years). The classic mechanism of injury is usually a child who falls on an outstretched upper extremity and suffers a hyperextension type injury at the elbow. Patients typically will have significant elbow pain and swelling. The distal fragment usually lies posterior, and this is best seen on lateral radiographs by drawing an imaginary line along the cortex of the anterior humerus and visualizing the capitellum lying posterior to this line (In the normal elbow, the anterior humeral line should bisect the capitellum). The posterior fat pad may also be present (always an abnormal finding) as well as an anterior displaced anterior fat pad ("sail sign"), both of which are indicative of a joint effusion.

Prompt diagnosis is critical owing to the potential complications of malunion and injury to the neurovascular structures that lie in close proximity, namely the brachial artery, median, radial, and ulnar nerves. The quality of the brachial and radial pulses should be assessed, as well as for the presence of cool, pale distal extremities and decreased capillary refill. The nerves can be quickly assessed by having the patient form a "thumbs up" sign (radial nerve), tight fist covering thumb (median nerve), and the "OK sign" by having them approximate the thumb and index finger (anterior interosseous nerve). It is also important to assess for signs of compartment syndrome, including increasing pain on passive extension of the fingers, parasthesias, pallor, pulselessness, or paralysis.

Supracondylar fractures are typically designated as Type I, II, or III depending on the amount of displacement. Type I fractures are non-displaced and may be treated conservatively in a long arm cast with elbow held in at least $90^{\circ}$ of flexion for 3-4 weeks. Type II fractures are partially displaced but have the posterior periosteal hinge intact, while Type III fractures are completely displaced with no contact between the two fragments. Both Type II and Type III fractures usually require closed reduction with percutaneous $\mathrm{K}$ wire insertion to hold normal position. Operative reduction is usually required if rotational deformity is noted, significant medial comminution exists, or if the reduction cannot be held in $100-120^{\circ}$ of flexion. Volkmann's ischemia is a dreaded complication, which can occur if a displaced fracture is treated with a cast in hyperflexion. The restriction that the cast provides coupled with extensive swelling can cause permanent neurovascular damage [11].

\section{Nursemaid's elbow}

Nursemaid's elbow, more accurately termed annular ligament displacement (ALD), is the most common upper 
extremity injury presenting to emergency departments in children less than 6 years of age. Patients usually will complain of elbow pain and parents may notice the sudden decreased movement of that arm.

ALD occurs when the annular ligament is displaced in between the humeral capitellum and the radial head. This is usually caused by an abrupt axial traction placed on the forearm while the elbow is fully extended and forearm pronated.

These injuries can usually be easily corrected by simple reduction techniques, involving either a supination/flexion sequence or a hyperpronation technique. Classically, the supination/flexion technique has been utilized by most practitioners, but studies seem to indicate that the hyperpronation technique may be more effective than supination/ flexion on first attempt and may be associated with less discomfort. One study involving several pediatric emergency departments randomly assigned patients presenting with findings suggestive of ALD to either the flexion/ supination or hyperpronation technique [12]. A total of 85 patients were included in the study and $95 \%$ of those randomized to the hyperpronation group had successful reduction on first attempt compared to $77 \%$ successful reduction in the supination/flexion group. The hyperpronation group also required fewer attempts at successful reduction than did the flexion/supination group, with overall success rate of the hyperpronation group being $97.5 \%$ compared to $86 \%$ in the supination/flexion group.

Another prospective study indicated that the forced pronation technique was perceived as being less painful than the supination-flexion technique by both nurses and parents [13].

\section{Conclusion}

When evaluating a pediatric or adolescent with elbow pain it is important to consistently take a thorough history and accurate physical exam. It should first be determined if pain is acute or chronic. If acute, serious fractures, including supracondylar fractures should be ruled out, as these usually need prompt orthopaedic referral. Mechanism of injury, or the types of activities that exacerbate the pain should also be elucidated. For the chronic, overuse types of injuries that cause elbow pain, determining what part of the throwing interval can be very helpful. Third, the location of the pain can also be of use, especially for the chronic types of elbow pain. The physical exam can further delineate the pathology, and close attention should be paid to range of motion, or lack thereof and the location of maximal tenderness. Conventional radiographs can then be used, and sometimes further imaging modalities may be necessary. It is vitally important to know the sequence of appearance of the secondary ossification centers when evaluating these images. By following these steps in comprehensive and systemic manner, the evaluation of pediatric elbow pain can be done confidently and accurately.

Acknowledgement The author wishes to thank Dr. James Andrews, Dr. Lyle Cain, Dr. Tracy Ray, and Dr. Jason Robertson for their advice and assistance with this project.

Open Access This article is distributed under the terms of the Creative Commons Attribution Noncommercial License which permits any noncommercial use, distribution, and reproduction in any medium, provided the original author(s) and source are credited.

\section{References}

1. Hutchinson MR, Ireland ML. Overuse and throwing injuries in the skeletally immature athlete. Instr Course Lect. 2003;52:2536.

2. Kaeding CC, Whitehead R. Musculoskeletal injuries in adolescents. Primary Care. 1998;25(1):211-23. March.

3. Bradley JP, Petrie RS. Osteochondritis dissecans of the humeral capitellum, diagnosis and treatment. Clin Sports Med. 2001;20(3):565-90.

4. Jackson D, Silvino N, Reimen P. Osteochondritis in the female gymnast's elbow. Arthroscopy. 1989;5:129-36.

5. Schenk RJ, Athanasiou K, Constantinides G, et al. A biomechanical analysis of articular cartilage of the human elbow and potential relationship to osteochondritis dissecans. Clin Orthop. 1994;299:305-12.

6. Stubbs MJ, Field LD, Savoie FH. Osteochondritis dissecans of the elbow. Clin Sports Med. 2001;20(1):1-9.

7. Haraldson S. Acta Orthop Scand. 1959;38(suppl):1-232.

8. Takahara M, Shundo M, Kondo M, et al. J Bone Joint Surg Am. 1998;80:892-7.

9. Herring JA. Upper extremity fractures. (2002). In: Tachdigan's pediatric orthopaedics, 3rd ed. Philadelphia: WB Saunders Co. p. 2115-2250.

10. Hart ES, Grottkau BE, Rebello GN, Albright MB. Ortho Nurs. 2006;25(5):311-25. Sept.

11. Beaty JH, Kasser JR. The elbow region: general concepts in the pediatric patient. In: Rockwood CA, Wilkins B, editors. Fractures in children. 5th ed. Philadelphia: Lippincott Williams and Wilkins; 2001.

12. Macias CG, Bothner J, Wiebe R. Pediatrics. 1998;102(1):e10. Jul.

13. Green DA, et al. Pediatr Emerg Care. 2006;22(4):235-8. Apr. 